\title{
Pyroelectric Crystal Generated Very Low Dose X-ray Radiation Enhanced NQO1 Upregulation and Apoptotic Cell Death in Beta-Lapachone Treated NQO1-Deficient LNCaP Prostate Cancer Cells
}

\author{
Saheed Oluwasina Oseni ${ }^{1, *}$, James Kumi-Diaka ${ }^{1}$, Rolando Branly ${ }^{2}$, Joubin Jebelli 1 , \\ Elsa Quiroz ${ }^{1}$
}

1

Department of Biological Sciences, Florida Atlantic University, 3200 College Avenue, Davie, FL 33314, USA; E-Mails: jdiaka@fau.edu (J.K.-D.); jjebelli@fau.edu (J.J.); elsqui@hotmail.com (E.Q.)

2 Department of Physical Sciences, Broward College, 3501 Davie Rd, Davie, FL 33314, USA;

E-Mail: rbranly@gmail.com

* Author to whom correspondence should be addressed; E-Mail: soseni2013@fau.edu; Tel.: +1-786-4755-746.

Received: 29 September 2015 / Published: 16 October 2015

\begin{abstract}
An estimated 220,800 new prostate cancer cases and 27,540 deaths are expected to occur in US men by the end of 2015. Beta-lapachone ( $\beta$-lap) is a promising anticancer bioreductive ortho-naphthoquinone. Recent studies suggest that $\beta$-lap kills cancer cells by targeting the $\mathrm{NAD}(\mathrm{P}) \mathrm{H}$ : quinone oxidoreductase (NQO1) enzyme levels in cancer tissues. But LNCaP, a PCa cell line is deficient in NQO1 enzyme levels. In this study, the potential role of very low doses of $\mathrm{x}$-ray radiation (VLDR) from a portable pyroelectric generator at $20 \mathrm{mGy} / \mathrm{hr}$ in enhancing the chemopreventive effects of $\beta$-lap in LNCaP PCa cells were investigated. The combination treatment-induced cytotoxicity was investigated via MTT and Trypan blue exclusion assays. Dicoumarol (an NQO1 inhibitor) was co-administered to assess the effect of VLDR on NQO1 modulation. NBT assay was used to assess the intracellular ROS levels. Fluorescence microscopy was also used to assess the mode of cell death. The data suggests that VLDR induced a rise in ROS levels, followed by upregulation in NQO1 levels. The major mode of cell death by this combination therapy was found to be via Apoptosis. In conclusion, our results confirm that VLDR-induced NQO1 levels contribute to cell death.
\end{abstract}


Keywords: prostate cancer; apoptosis; beta-lapachone; pyroelectricity; reactive oxygen species; NQO1

\section{Introduction}

Prostate cancer $(\mathrm{PCa})$ remains the most commonly diagnosed non-cutaneous cancers and the second leading cause of cancer death in American men. In 2015 alone, an estimated 220,800 men was diagnosed with new cases of prostate cancer and an estimated 27,540 deaths are expected. This is in addition to the over 2.5 million men battling prostate cancer in the United States currently (SEER, 2015). The explicit amalgamation of two or more cancer therapeutic modalities such as surgery, radiation, and chemotherapy, has been one of the main interests of clinical investigation for several decades. Betalapachone ( $\beta$-lap) is a bioreductive ortho naphthoquinone (Kumi-Diaka, 2002). The anticancer activities of $\beta$-lap have been correlated with the enzyme activity of NQO1 (Kumi-Diaka et al., 2004). This means that NQO1+ cells would be more sensitive to $\beta$-lap induced cytotoxicity compared to NQO1 deficient cancer cells. A prior study has shown that a very low dose of X-radiation (VLDR) from pyroelectric generator at a dose of $20 \mathrm{mGy} / \mathrm{hr}$. is able to enhance the chemopreventive effects of genistein isoflavone (Oseni et al., 2014). The aim of this study was to investigate the enhancing effects of VLDR derived from a portable pyroelectric generator on the chemopreventive and/or chemotherapeutic effects of $\beta$-lap in NQO1 deficient LNCaP prostate cancer cells $(\mathrm{PCa})$ in vitro.

\section{Result and Discussion}

We observed a significantly decrease $(P<0.05)$ in intracellular ROS generation in LNCaP cells exposed to VLDR after 24 hours of incubation, though this was found to be time-dependent in which it's level depreciates with time. The cytotoxicity effect of the combination treatments was found to be more pronounced $(P<0.05)$ relative to treatment with VLDR or $\beta$-lap alone. In addition, a dose-dependent response was also observed, in which more cells were affected as the dose of $\beta$-lap was increased. However, when compared to the single treatment alone at the same dose and time, the cytotoxicity was found to be higher at lower doses $(1.0$ and $2.0 \mu \mathrm{M})$ compared to high doses $(5.0$ and $7.0 \mu \mathrm{M})$. The initial increase in the ROS levels may be responsible for the activation of the ROS-Nrf1/2-Keap-ARE pathway, which result in the downstream upregulation of NQO1 in the LNCaP cells. Co-administration of $\beta$-lap and dicoumarol, did not result to any significant change $(P>0.05)$ in sensitivity in LNCaP cells exposed to $\beta$-lap alone, but result in a significant decrease $(P<0.05)$ in VLDR $+\beta$-lap combined treatment. 

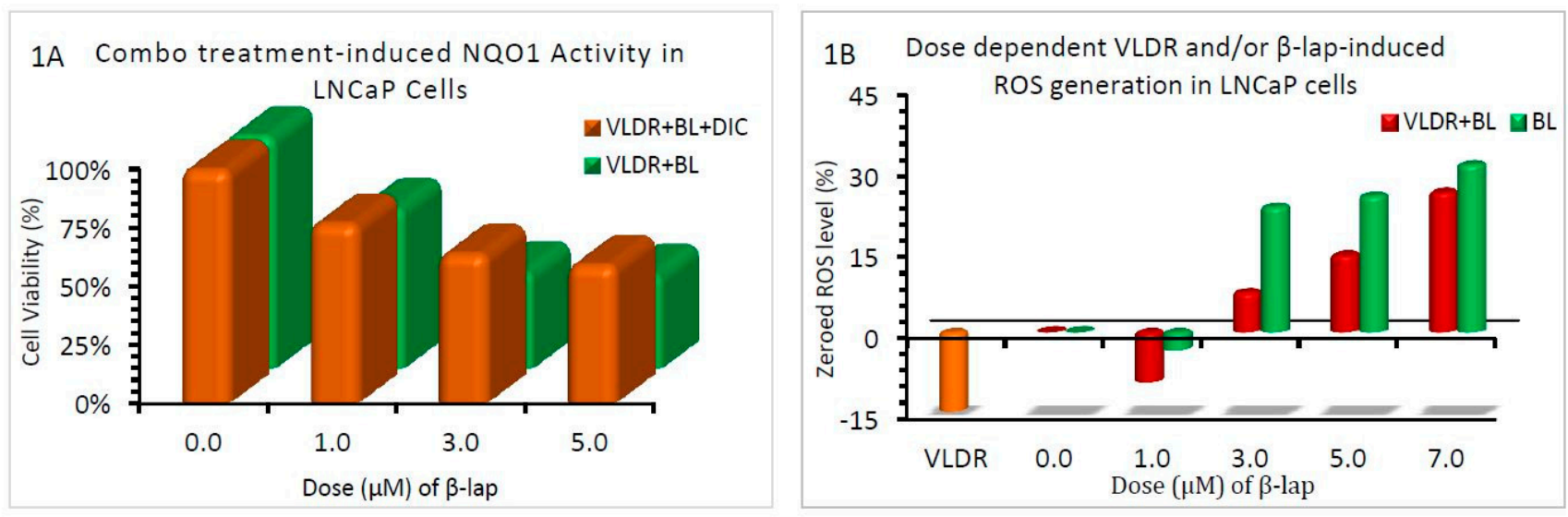

Figure 1. (A) Showing NQO1 activity in LNCaP cells post treatment with VLDR+ BL0-7. DIC @ $50 \mu \mathrm{M}$ dose, an NQO1 inhibitor was co-administered with the combo treatment to inhibit the activity of NQO1 post treatment. There was dose-dependent significant difference $(P<0.05)$ as a result of co-administration of DIC with the combo regimen. (B) Bar-chart representing the dose-dependent treatment-induced intracellular ROS levels in LNCaP cells exposed to both VLDR and/or BL. ROS readings was taken 24 hours post treatment.
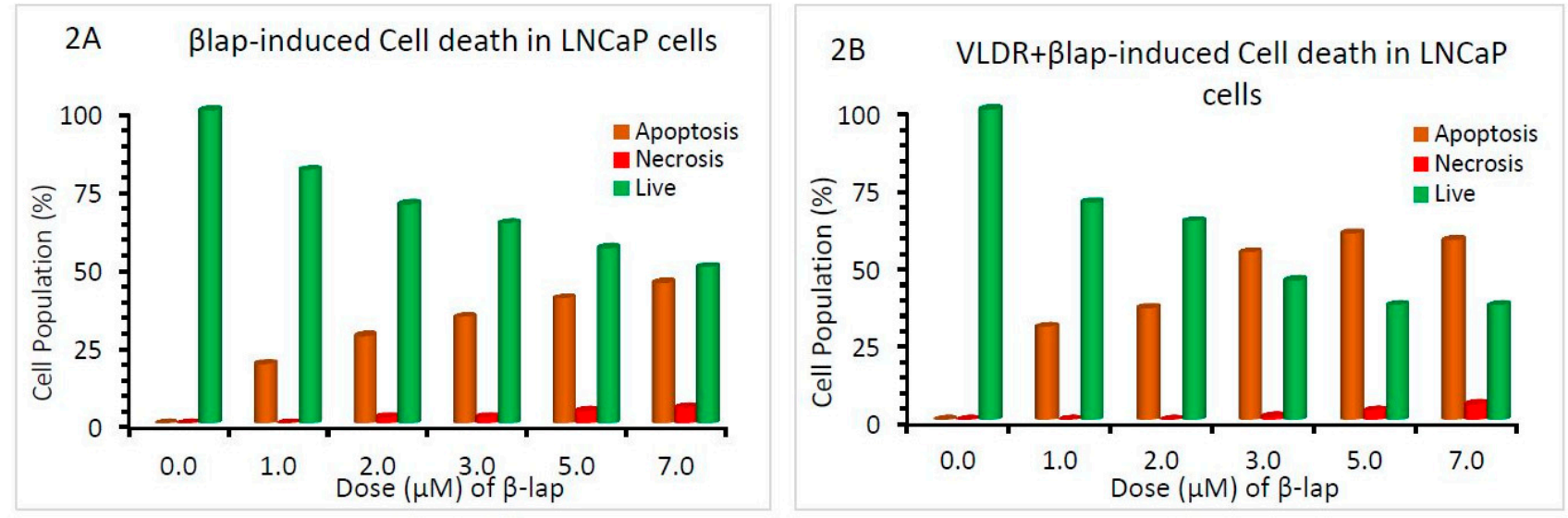

Figure 2. (A,B) Acridine orange/ ethidium bromide double fluorescence staining was used to distinguish viable (live), apoptotic and necrotic cells based on green or orange-red fluorescence staining respectively and by their cell morphology. The major mode of cellular death in all treatment groups was apoptosis, with the highest percentage $(P<0.05)$ observed in LNCaP cell lines with VLDR $+\beta$-lap combination treatments. There was significant difference $(P<0.05)$ in the apoptotic cell death observed in single and combined treatments. 

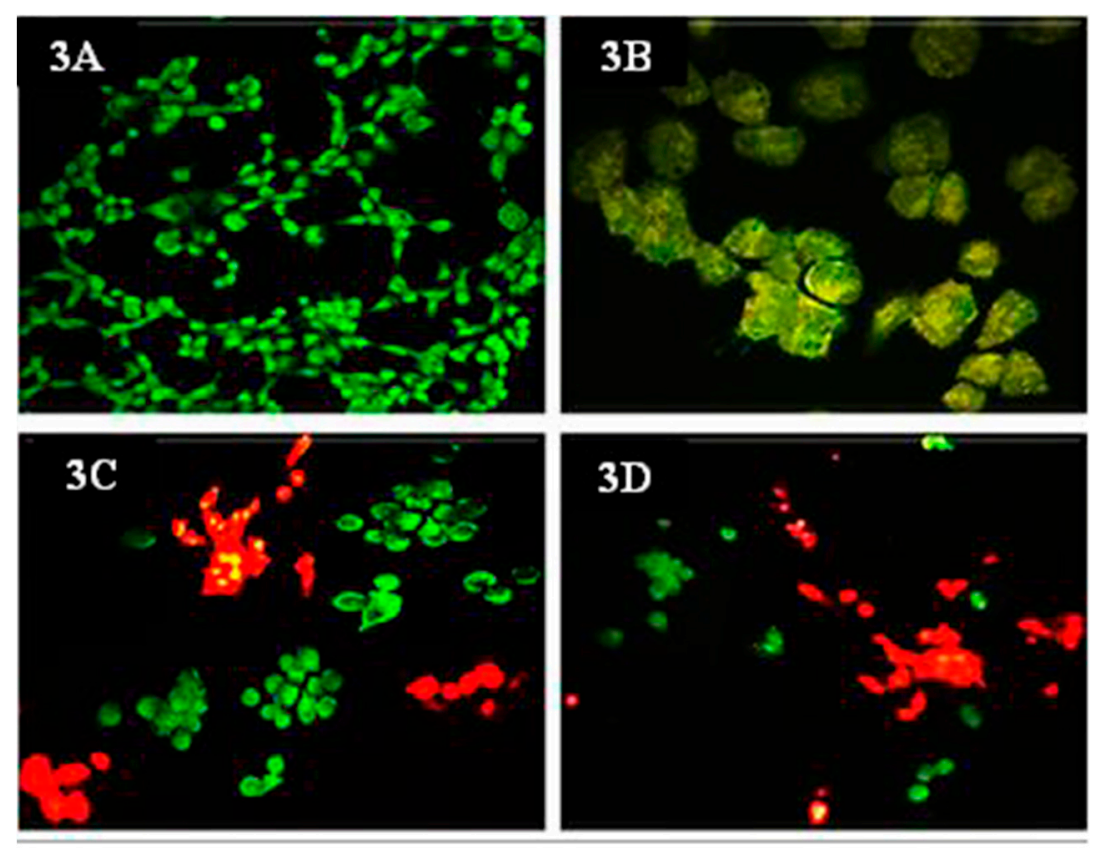

Figure 3. (A-D) Showing micrographs of acridine orange/ethidium bromide double fluorescence staining. (3A) is showing untreated cells with green fluorescence. (3B) is showing cells exposed to VDLR; the yellowish green fluorescence is representing cells undergoing either autophagy or early stage apoptotic response; (3C) and (3D) are showing cells treated with $\beta$-lap (BL) only and VLDR+ $\beta$-lap respectively, greater percentage of the cells in (3C) are resistant (green) compared to (3D) and more cells are apoptotic (red fluorescence) compared to Figure 3C.

\section{Materials and Methods}

NQO1-deficient LNCaP PCa cell line was initially cultured to $70-80 \%$ confluence, and thereafter seeded $\left(1 \times 10^{4}\right.$ cells per well $)$ in 92 wells microplate. All irradiations were performed using a miniaturized portable pyroelectric x-ray generator. Cells were irradiated by exposure to single doses of very low dose ionizing radiation (VLDR) at $20 \mathrm{mGy} / \mathrm{hr}$ (i.e. $0.3 \mathrm{mGy} / \mathrm{min}$ dose rate) low LET X-ray radiation and thereafter treated with different doses $(0-7 \mu \mathrm{M})$ of $\beta$-lap. After exposure to single and combined treatment modes, cell viability and cell inhibition rates were determined using bioassays such as MTT and Trypan blue assays. ROS generation activity was also determined, and also for both the single and combined treatments using NBT assay. Dicoumarol, an NQO1 inhibitor was used to measure the NQO1 activity in LNCaP cells following single and combination treatments. Double fluorescence staining with acridine orange and ethidium bromide was used to determine major mode of treatment induced cell death.

\section{Conclusion}

The results from this present study strongly suggest that NQO1 activity in tumors may be selectively elevated using pyroelectrically generated very low dose radiation therapy to reduce resistance and/or to improve the cytotoxicity effects of $\beta$-lap against NQO1-deficient prostate cancer cells. 


\section{Author Contributions}

This work was carried out in collaboration between all authors. S.O.O participated in study design, data collection, data analysis and interpretation, did literature search and drafted/edited/revised manuscript, J.K-D and R.B participated in study design, data interpretation and revising of manuscript. J.J and E.Q assisted in data collection and lab protocols. All authors read and approved the final manuscript.

\section{Conflicts of Interest}

The authors declare no conflict of interest.

\section{References}

1. Surveillance Epidemiology and End Result (SEER). Stat Fact Sheets: Prostate Cancer. Available online: http://seer.cancer.gov/statfacts/html/prost.html (accessed on 13 October 2015).

2. Kumi-Diaka, J. Chemosensitivity of human prostate cancer cells PC3 and LNCaP to genistein isoflavone and $\beta$-lapachone. Biology of the Cell 2002, 94, 37-44.

3. Kumi-Diaka, J.; Saddler-Shawnette, S.; Aller, A.; Brown, J. Potential mechanism of phytochemicalinduced apoptosis in human prostate adenocarcinoma cells: Therapeutic synergy in genistein and beta-lapachone combination treatment. Cancer Cell International. 2004, 4, 1-5.

4. Oseni, S.O.; Kumi-Diaka, J.; Branly, R; Jebelli, J. Pyroelectrically Generated Very Low Dose Ionizing Radiation Potentiates the Chemotherapeutic and Chemopreventive Effects of Genistein Isoflavone in Human Prostate Cancer Cells". Journal of Cancer Prevention \& Current Research. 2014, 1, 14-20.

(C) 2015 by the authors; licensee MDPI, Basel, Switzerland. This article is an open access article distributed under the terms and conditions of the Creative Commons Attribution license (http://creativecommons.org/licenses/by/4.0/). 